Published in final edited form as:

Lancet Respir Med. 2019 February ; 7(2): 154-162. doi:10.1016/S2213-2600(18)30418-1.

\title{
Treatment with allogeneic mesenchymal stromal cells for moderate to severe acute respiratory distress syndrome (START study): a randomised phase 2 a safety trial
}

\author{
Michael A Matthay, Carolyn S Calfee, Hanjing Zhuo, B Taylor Thompson, Jennifer G \\ Wilson, Joseph E Levitt, Angela J Rogers, Jeffrey E Gotts, Jeanine P Wiener-Kronish, \\ Ednan K Bajwa, Michael P Donahoe, Bryan J McVerry, Luis A Ortiz, Matthew Exline, John \\ W Christman, Jason Abbott, Kevin L Delucchi, Lizette Caballero, Melanie McMillan, David \\ H McKenna, Kathleen D Liu \\ Department of Medicine and Anesthesia (Prof M A Matthay MD, Prof C S Calfee MD, J E Gotts \\ MD, Prof K D Liu MD), Department of Psychiatry (Prof K L Delucchi PhD), Bone and Marrow \\ Transplant Laboratory (L Caballero MS, M McMillan BS), and Cardiovascular Research Institute \\ (Prof M A Matthay, Prof C S Calfee, H Zhuo MPH, J Abbot BS, K D Liu), University of California, \\ San Francisco, CA, USA; Department of Medicine (Prof B T Thompson MD, E K Bajwa MD) and \\ Department of Anesthesiology (Prof J P Wiener-Kronish MD), Massachusetts General Hospital, \\ Harvard Medical School, Boston, MA, USA; Department of Emergency Medicine (J G Wilson MD) \\ and Department of Medicine (J E Levitt MD, A J Rogers MD), Stanford University, Stanford, CA, \\ USA; Department of Medicine (Prof M P Donahoe MD, B J McVerry MD) and Department of \\ Environmental and Occupational Health (Prof $L A$ Ortiz MD), University of Pittsburgh, Pittsburgh, \\ PA, USA; Department of Medicine, Ohio State University Medical Center, Columbus, OH, USA (M \\ Exline MD, Prof J W Christman MD); and University of Minnesota, Molecular and Cellular \\ Therapeutics, Saint Paul, MN, USA (Prof D H McKenna MD)
}

\section{Summary}

\begin{abstract}
Background-Treatment with bone-marrow-derived mesenchymal stromal cells (MSCs) has shown benefits in preclinical models of acute respiratory distress syndrome (ARDS). Safety has not been established for administration of MSCs in critically ill patients with ARDS. We did a phase 2a trial to assess safety after administration of MSCs to patients with moderate to severe ARDS.
\end{abstract}

Methods-We did a prospective, double-blind, multicentre, randomised trial to assess treatment with one intravenous dose of MSCs compared with placebo. We recruited ventilated patients with

\footnotetext{
Correspondence to: Prof Michael A Matthay, Departments of Medicine and Anesthesia, Cardiovascular Research Institute, University of California, San Francisco, M-917, San Francisco, CA 94143, USA michael.matthay @ucsf.edu.

Contributors

MAM, CSC, HZ, BTT, JGW, JEG, LAO, KLD, LC, MM, DHM, and KDL designed the study design and developed the protocol. MAM, CSC, BTT, JEL, AJR, JEG, JPW-K, EKB, MPD, BJM, ME, JWC, and KDL were responsible for study enrolment and data collection. MAM, CSC, HZ, JEG, JA, and KDL were responsible for biorepository management and biomarker analyses. MAM, CSC, $\mathrm{HZ}$, JEG, KLD, and KDL analysed the data and wrote the report. All authors revised the report and read and approved the final version before submission.

Data sharing

No additional data are available for this Article.

See Online for appendix
} 
moderate to severe ARDS (ratio of partial pressure of oxygen to fractional inspired oxygen $<27$ $\mathrm{kPa}$ and positive end-expiratory pressure [PEEP] $\geq 8 \mathrm{~cm} \mathrm{H}_{2} \mathrm{O}$ ) in five university medical centres in the USA. Patients were randomly assigned 2:1 to receive either $10 \times 10^{6} / \mathrm{kg}$ predicted bodyweight MSCs or placebo, according to a computer-generated schedule with a variable block design and stratified by site. We excluded patients younger than 18 years, those with trauma or moderate to severe liver disease, and those who had received cancer treatment in the previous 2 years. The primary endpoint was safety and all analyses were done by intention to treat. We also measured biomarkers in plasma. MSC viability was tested in a post-hoc analysis. This trial is registered with ClinicalTrials.gov, number NCT02097641.

Findings-From March 24, 2014, to Feb 9, 2017 we screened 1038 patients, of whom 60 were eligible for and received treatment. No patient experienced any of the predefined MSC-related haemodynamic or respiratory adverse events. One patient in the MSC group died within $24 \mathrm{~h}$ of MSC infusion, but death was judged to be probably unrelated. 28-day mortality did not differ between the groups (30\% in the MSC group vs $15 \%$ in the placebo group, odds ratio $2.4,95 \%$ CI 0.5-15.1). At baseline, the MSC group had numerically higher mean scores than the placebo group for Acute Physiology and Chronic Health Evaluation III (APACHE III; 104 [SD 31] vs 89 [33]), minute ventilation (11.1 [3.2] vs 9.6 [2.4] L/min), and PEEP (12.4 [3.7] vs $10 \cdot 8$ [2.6] cm $\mathrm{H}_{2} \mathrm{O}$ ). After adjustment for APACHE III score, the hazard ratio for mortality at 28 days was 1.43 (95\% CI $0 \cdot 40-5 \cdot 12, \mathrm{p}=0 \cdot 58$ ). Viability of MSCs ranged from $36 \%$ to $85 \%$.

Interpretation-One dose of intravenous MSCs was safe in patients with moderate to severe ARDS. Larger trials are needed to assess efficacy, and the viability of MSCs must be improved.

Funding-National Heart, Lung, and Blood Institute.

\section{Introduction}

Acute respiratory distress syndrome (ARDS) is associated with protein-rich pulmonary oedema and acute respiratory failure characterised by acute inflammation and injury to the lung and epithelia. ${ }^{1,2}$ In a multinational observational study, ARDS occurred in $10 \%$ of patients admitted to intensive-care units, and mortality among these patients was increased by more than $30 \%$ despite improvements in supportive care and ventilator management. ${ }^{3}$

In several preclinical studies using animal models of ARDS, treatment with mesenchymal stromal cells (MSCs) derived from bone marrow, umbilical cord, or adipose tissue has reduced the severity of acute lung injury from infectious and non-infectious causes. ${ }^{4,5}$ In a $24 \mathrm{~h}$ model of severe lung injury from bacterial pneumonia in sheep, human bone-marrowderived MSCs improved oxygenation and reduced extravascular lung water compared with a placebo control, without adverse haemodynamic effects or respiratory compromise. ${ }^{6}$ In addition, allogeneic MSCs derived from human bone marrow were effective in models of acute lung injury in ex-vivo perfused human lung preparations injured with endotoxin or Gram-negative bacteria. ${ }^{7,8}$ In some studies the MSCs were administered intravenously and in others they were administered by the intratracheal or intrabronchial routes. The preclinical data overall indicate reduced lung vascular and epithelial permeability to protein, increased clearance of alveolar oedema fluid, and anti-inflammatory and antimicrobial properties, which support the therapeutic potential of bone-marrow-derived MSCs for ARDS. ${ }^{9}$ 
Several clinical trials have suggested that patients with moderate to severe ARDS (ratio of partial pressure of oxygen to fractional inspired oxygen $\left[\mathrm{PaO}_{2}: \mathrm{FiO}_{2}\right]<27 \mathrm{kPa}$ ), in whom lung inflammation and diffuse alveolar damage are increased, ${ }^{10}$ have differential and greater responses to treatments than patients with mild ARDS $\left(\mathrm{PaO}_{2}: \mathrm{FiO}_{2} 27-40 \mathrm{kPa}\right)$. Given this difference in response, the increased mortality risk in patients with moderate to severe ARDS (29-48\%), ${ }^{11}$ and the multipotent effects of cell-based therapies, ${ }^{12}$ we tested MSCbased treatment in patients with moderate to severe ARDS.

As a first step in testing safety, we did a phase 1, open-label, dose-escalation trial in nine patients with moderate to severe ARDS, using 1, 5, and $10 \times 10^{6} \mathrm{MSCs} / \mathrm{kg}$ predicted bodyweight. Infusions at all doses were well tolerated. ${ }^{13}$ Therefore, we obtained approval from the US Food and Drug Administration (FDA) to do a randomised, double-blind, placebo-controlled, phase 2a trial to test the safety of intravenous treatment with the highest dose in patients with moderate to severe ARDS.

\section{Methods}

\section{Study design and patients}

START was a double-blind, randomised, placebo-controlled, phase 2a trial that was done in five university medical centres in the USA (University of California San Francisco, Stanford University, University of Pittsburgh Medical Center, Ohio State University, and Massachusetts General Hospital). Eligible patients were endotracheally intubated, had a $\mathrm{PaO}_{2}: \mathrm{FiO}_{2}$ less than $27 \mathrm{kPa}$, were mechanically ventilated with at least $8 \mathrm{~cm} \mathrm{H}_{2} \mathrm{O}$ positiveend expiratory pressure (PEEP), had bilateral pulmonary infiltrates consistent with pulmonary oedema on chest radiographs, and had no clinical evidence of left-heart failure or volume overload as the primary cause of the pulmonary oedema. A protocol amendment was made to allow enrolment of patients with PEEP of $5 \mathrm{~cm} \mathrm{H}_{2} \mathrm{O}$ if they had evidence of barotrauma.

Exclusion criteria (appendix) included age younger than 18 years, ARDS present for more than $96 \mathrm{~h}$, pregnancy or breastfeeding, being an inmate in the prison system, having received treatment for cancer in the past 2 years (except non-melanoma skin cancer), having an underlying medical status with life expectancy less than 6 months, moderate to severe liver disease (Child-Pugh score $>12$ ), severe chronic lung disease with the use of home oxygen, or partial arterial pressure of carbon dioxide greater than $7 \mathrm{kPa}$, and not being committed to full support (ie, had do not resuscitate or limit on life support orders).

The trial was approved by the institutional review boards at each medical centre, the FDA (15331), and the National Heart, Lung, and Blood Institute (U01HL108713). The clinical protocol is available in the appendix. A data safety monitoring board approved the trial design and reviewed serious adverse events and the results after 20 and 40 patients had been enrolled. Written informed consent was obtained from patients or authorised surrogates.

\section{Randomisation and masking}

Patients were randomly assigned (2:1) to receive either $10 \times 10^{6} \mathrm{MSC} / \mathrm{kg}$ predicted bodyweight or placebo (Plasma-Lyte A, Baxter, Deerfield, IL, USA). The randomisation had 
a variable block design, was stratified by site, and the sequence was generated by computer. The allocation sequence could be accessed by each cell laboratory through a dedicated website. Personnel in the cell laboratories were not masked, but patients, clinical staff, and investigators were unaware of treatment assignment. To maintain masking of the investigators and clinicians, bags containing the study products and intravenous tubing had opaque coverings applied in the cell laboratories. No measures were taken to check the success of masking. For data analysis, investigators were unaware of treatment allocation until the database was fully cleaned and locked.

\section{Procedures}

Allogeneic MSCs derived from human bone marrow were prepared from three donors (aged 18-45 years, one woman and two men) with support from the National Heart, Lung, and Blood Institute Production Assistance for Cellular Therapies Program at the University of Minnesota (Minneapolis, MN, USA). MSCs were manufactured from fresh (four lots) or cryopreserved (eight lots) bone marrow. The mononuclear cell fraction was enriched with Ficoll. Mononuclear cells were tested for nucleated cell count, differential, viability, flow cytometry (CD105, CD73, CD90, CD45, CD34, CD14, CD19, and HLA-DR), and sterility before seeding at 100000 cells $/ \mathrm{cm}^{2}$ for culture. At 70\% confluence, MSCs were lifted and passaged at a low density $\left(40-50\right.$ cells $\left./ \mathrm{cm}^{2}\right)$ into 24 cell factories. At $70-80 \%$ confluence, the MSCs were harvested, washed, resuspended, and cryopreserved. Karyotyping (G banding) was done for all lots of MSCs and was normal. The criterion for release of MSCs from the University of Minnesota was at least $70 \%$ viability.

The cryopreserved MSCs were shipped to the clinical sites frozen at a maximum temperature of $-150^{\circ} \mathrm{C}$, in a validated liquid nitrogen dry shipper with a continuous temperature monitoring device. Upon receipt, the cellular product was inspected and stored in a controlled, continuously monitored liquid nitrogen storage tank. Before administration, the MSCs were thawed, washed to remove dimethyl sulfoxide (DMSO), and resuspended in Plasma-Lyte A by a local cell therapy laboratory. DMSO was removed because studies in sheep with Pseudomonas aeruginosa pneumonia suggested that efficacy of MSCs would be decreased without this step before intravenous administration. ${ }^{6}$ The total volume of the MSC or placebo infusion was $100 \mathrm{~mL}$. Viability of infused MSCs was determined by trypan blue exclusion after the MSCs had been thawed and prepared for infusion at each of the clinical sites. The time to measurement of viability after MSC preparation was approximately $1 \mathrm{~h}$, although no time range was specified in the standard operating procedures.

After a patient had been clinically stable for $2 \mathrm{~h}$ (transcutaneous oxygen saturation 88-95\% without the need for $\mathrm{FiO}_{2}>0.8$ or PEEP $>20 \mathrm{~cm} \mathrm{H}_{2} \mathrm{O}$ and use of maximum two vasopressors, $<0 \cdot 1 \mu \mathrm{g} / \mathrm{kg}$ per min epinephrine, or both, for blood pressure support) in the supine position, infusion was started with a standard blood filter tubing set with a pore size of $170 \mu \mathrm{m}$. The study product was infused by gravity over $60-80 \mathrm{~min}$. The infusion rate, based on the droplet count, was controlled by a physician investigator who remained at the patient's bedside for the duration of the infusion and in the intensive care unit for $6 \mathrm{~h}$ after the start of infusion to monitor for any signs of adverse reaction. All patients were ventilated according to the modified ARDS Network lower tidal volume protocol. ${ }^{14}$ 
Theoretically, MSC infusion could increase pulmonary vascular resistance in the presence of injured pulmonary microcirculation, potentially by aggregating or clumping in capillaries or small arterioles. Subsequent potential risks are acute right heart failure, impaired cardiac output, and haemodynamic instability. Additionally, infusion of MSCs could potentially worsen ventilation-perfusion mismatch and result in further impairment of oxygenation or carbon dioxide excretion. All patients, therefore, were monitored closely for any changes in a prescribed list of respiratory or cardiovascular parameters (panel). The incidence and nature of all serious adverse events were reviewed and independently assessed by the data safety monitoring board to determine whether they were related to MSC administration, with special focus on events that would be unexpected in a critically ill patient with ARDS. Additionally, we measured creatinine, total bilirubin, and alanine aminotransferase concentrations in serum on days 3, 7, and 14 after infusion if patients were still in hospital. Methods for data collection and in-study measurements are described elsewhere. ${ }^{15}$

\section{Clinical and biological outcomes}

The primary outcome of the study was the safety of the MSC infusion, assessed with prespecified infusion-associated adverse events focused on acute haemodynamic or respiratory compromise (panel). The secondary outcomes were all-cause mortality at day 28 and day 60 , number of ventilator-free days to day 28 , duration of ventilation in patients alive at day 28 , number of intensive-care-free days to day 28 , number of days free from organ failure to day 28 (cardiovascular, coagulation, hepatic, and renal), and the sequential organ failure assessment (SOFA) score. ${ }^{16}$ For respiratory parameters, we calculated the oxygenation index with the following validated measure of respiratory function, ${ }^{17,18}$

$$
\text { oxygenation index }=\frac{\mathrm{FiO}_{2}(\%) \times \text { mean airway pressure }}{\mathrm{PaO}_{2}}
$$

and with the lung injury score. ${ }^{19}$ Biological markers of endothelial injury (angiopoietin 2), inflammation (interleukin [IL]-6 and IL-8), and alveolar epithelial injury (receptor for advanced glycation end products [RAGE]) were measured in plasma collected at baseline, 6 $\mathrm{h}$, and $24 \mathrm{~h}$ after infusion, and were measured with ELISA kits (R\&D Systems, Minneapolis, MN, USA).

\section{Post-hoc study of MSC viability}

After the trial was completed, MSC viability after thawing was found to be variable. Therefore, the University of California, San Francisco bone marrow transplant laboratory assessed the effects of washing to remove DMSO between thawing and resuspension of the MSCs in Plasma-Lyte A for infusion compared with simple thawing and dilution. We thawed MSCs that had originally been prepared for the trial but were not used. We measured the proportions of viable MSCs with trypan blue exclusion at 0, 30, 60, 120, and $180 \mathrm{~min}$ after removal from cryopreservation to simulate the time from before thawing to completing clinical infusion. 


\section{Statistical analysis}

Because safety was the primary endpoint, we did not calculate sample size on the basis of an efficacy endpoint. We therefore used the FDA's recommendation that 60 patients randomly assigned in a ratio of 2:1 would be enough to assess the primary endpoint. Analyses were done by intention to treat. Descriptive analyses of the variables were expressed as mean (SD), median (IQR), or number (\%). All-cause mortality at 28 days and 60 days was analysed with Cox proportional hazard models and reported as hazard ratios (HRs) with 95\% CIs. We also report HRs after adjustment for Acute Physiology and Chronic Health Evaluation III (APACHE III) scores to resolve imbalances in severity of illness at baseline. To investigate the effects of MSC treatment on biomarker concentrations in plasma, we used the Mann-Whitney-Wilcoxon test on the original scale or ANCOVA after log transformation to compare values in the MSC and placebo groups at baseline $(0 \mathrm{~h})$ with results at 6 and $24 \mathrm{~h}$ after the start of infusion. In a post-hoc analysis, MSC viability at the time of administration was separated into tertiles and biomarker changes within $24 \mathrm{~h}$ from the start of infusion were compared between tertiles and between the MSC and placebo groups.

Two-sided $\mathrm{p}$ values less than 0.05 were significant. All statistical analyses were done with Stata software, version 12.0. The independent data safety monitoring board reviewed safety data after 20 and 40 patients were enrolled. This trial is registered with ClinicalTrials.gov, number NCT02097641.

\section{Role of the funding source}

The funder of the study had no role in the study design, data collection, data analysis, data interpretation, or writing of this report. The corresponding author had full access to all the data in the study and had final responsibility for the decision to submit for publication.

\section{Results}

From March 24, 2014, to Feb 9, 2017, 1038 patients were screened for eligibility, of whom 975 were excluded, and 63 were randomly assigned to a treatment group (figure 1). Three patients were not eligible for treatment after randomisation and, therefore, 40 patients received MSCs and 20 patients received placebo (figure 1). All patients received their allocated treatments and were included in all outcome analyses.

Age, sex, and cause of ARDS were similar in the two groups at baseline (table 1). Scores were numerically higher in the MSC group than in the placebo group for severity of illness (SOFA and APACHE III) and respiratory parameters (minute ventilation and PEEP; table 1).

No patients had any of the prespecified adverse haemodynamic or respiratory safety events during study product infusion or within $6 \mathrm{~h}$ from the start of infusion (one-sided 97.5\% CI $0-0.09$ ). One patient in the MSC group had a fatal cardiopulmonary arrest $20 \mathrm{~h}$ after the start of product infusion and following a sustained fibreoptic bronchoscopy for clinical indications. This patient had a pre-existing history of coronary artery disease. The DSMB judged that the death was probably not related to the MSC infusion. All other deaths occurred after $24 \mathrm{~h}$, and most were attributable to multiorgan failure after withdrawal of life support. The 6-month and 12-month follow-up data are provided in the appendix. 
Mortality at 28 and 60 days was non-significantly higher in the MSC group than in the placebo group (table 2). The number of ventilator-free and organ-failure-free days were all numerically fewer in the MSC group than in the placebo group, but the differences were not significant (table 2). The number of intensive-care-free days was higher in the placebo group than in the MSC group (table 2). Further data on the prespecified secondary endpoints and the SOFA score at 3, 7, and 14 days are provided in the appendix. After adjustment for APACHE III score, the HRs for 28-day and 60-day mortality remained non-significant but moved towards 1 (figure 2).

The oxygenation index was numerically higher in the MSC group than in the placebo group at baseline (appendix). Over the following 2 days, oxygenation index in the MSC group reduced but not by significantly more than in the placebo group. A similar pattern in reduction of oxygenation index was seen in patients who remained intubated for 2 days (appendix).

At baseline, protein C concentration in plasma was numerically lower in the MSC group than in the placebo group, but no other biomarkers differed between groups at baseline (table 3). Concentrations of angiopoietin $2 \mathrm{had}$ reduced by $6 \mathrm{~h}$ after the start of infusion in the MSC group (figure 3). No changes from baseline were seen for IL-6, IL-8, RAGE, or protein $\mathrm{C}$ concentrations at 6 or $24 \mathrm{~h}$ in either group.

After completing the study, the viability of MSCs at the time of study product preparation was found to range widely from $36 \%$ to $85 \%$. Tertiles of viability were $36-56 \%$ ( $\mathrm{n}=14$ recipients), 57-69\% ( $\mathrm{n}=13)$, and 70-85\% ( $\mathrm{n}=13)$. In our post-hoc analysis of viabilitydependent effects on biomarkers, we found no differences for IL-6, IL-8, or RAGE at baseline (appendix p 5), but after adjustment for baseline biomarker values, concentrations of angiopoietin 2 decreased progressively, and in the intermediate and the highest tertiles of MSC viability were significantly lower than in the placebo group (appendix). In addition, oxygenation index seemed to improve from baseline to 2 days after the start of infusion in the intermediate and highest tertiles of MSC viability, albeit non-significantly (appendix). Further comparisons of secondary outcomes with the placebo group and between MSC tertiles are provided in the appendix.

Washing to remove DMSO during preparation was associated with a notable reduction in the proportion of viable MSCs compared with simple thawing (figure 4). We found no significant differences in MSC viability among the donors (appendix). MSC viability was higher at one preparation site than the other four (appendix), but we could identify no differences in processing of MSCs in the viability measurements.

\section{Discussion}

In this double-blind, randomised, placebo-controlled phase 2a trial, we found no MSCrelated predefined haemodynamic or respiratory adverse events within $6 \mathrm{~h}$ of the start of infusion in 40 patients with moderate to severe ARDS. One patient died within $24 \mathrm{~h}$ of receiving MSCs but the DSMB judged the death unlikely to be related to administration of the cell product.

Lancet Respir Med. Author manuscript; available in PMC 2020 October 30. 
Mortality at 28 and 60 days was numerically but not statistically higher in the MSC group than in the placebo group. The numerical difference might have been driven by imbalances in the severity of illness, represented by SOFA and APACHE III scores, at baseline. Several baseline respiratory variables (minute ventilation, respiratory rate, oxygenation index, and PEEP level) indicated that respiratory failure might have been more severe in the MSC group than in the placebo group. Although neither the unadjusted nor the adjusted analyses showed significant differences in the secondary outcomes, we recognise that the higher mortality in the MSC group could represent an unfavourable effect of MSCs. Nevertheless, mortality in the placebo group was lower than anticipated, ${ }^{12}$ whereas that in the MSC group was as anticipated. Additionally, drawing conclusions from this small clinical trial is difficult due to wide CIs for the secondary endpoints.

Post-hoc analysis indicated reductions in oxygenation index over the 2 days following infusion among patients who received MSCs compared with those who received placebo. This analysis is limited for several reasons, including death, extubation, and some missing data. We attempted to account for these effects by doing a paired analysis only in patients intubated during the 2 days after infusion, and the findings remained similar. However, the numerical improvement in oxygenation should not be overinterpreted as a favourable finding until it has been assessed further in a larger trial.

The decrease of concentrations of angiopoietin 2 in plasma was significantly greater in the MSC group than in the placebo group $6 \mathrm{~h}$ after the start of infusion. This decrease suggests a biological effect of the MSC treatment. Angiopoietin 2 is a well recognised mediator and biomarker of pulmonary ${ }^{20}$ and systemic vascular injury. ${ }^{21}$ We have found previously that angiopoietin-2 concentrations have important predictive value for the development of ARDS. ${ }^{22}$ In addition, they robustly predict poor clinical outcomes in adults ${ }^{23}$ and children ${ }^{24}$ with ARDS, and are recognised as pathogenetic and prognostic factors in patients with pneumonia. ${ }^{25}$ The reductions in angiopoietin- 2 concentrations in this trial might have been related to the release of anti-inflammatory factors that can moderate lung injury. ${ }^{5}$ No significant changes were seen in concentrations of IL-6, IL-8, RAGE, or protein C.

The most unanticipated finding in this trial, found only after study completion, was the wide range of MSC viability after preparation for infusion (36-85\%). Substantial variations had not been anticipated and, therefore, we planned no checks of viability during the trial. Because of this finding, we did a post-hoc analysis, which showed a significant viabilitydependent effect on angiopoietin- 2 concentrations in plasma $6 \mathrm{~h}$ after administration. No effect on angiopoietin 2 was seen in the lowest viability tertile, but significant differences between groups were seen in the intermediate $(\mathrm{p}=0.02)$ and highest tertiles $(\mathrm{p}=0.001)$. We also saw a slight improvement in oxygenation index on day 2 in the intermediate and highest viability tertiles. No other significant differences were seen, although there was a favourable shift of the adjusted HR for mortality in the higher MSC viability tertiles. Although the differences are inconclusive, they suggest that there is potential for improved efficacy of MSCs with adequate viability. We suggest two explanations for the variation in MSC viability in this trial. First, a study from the University of Minnesota, which has a centre that produces MSCs, showed $14 \%$ less eventual MSC viability by flow cytometry with cell products derived from cryopreserved rather than fresh bone marrow. ${ }^{26}$ Second, our 
procedure in the trial to remove the DMSO from the thawed MSCs also seemed to reduce MSC viability. The scientific justification for the wash step was based on unpublished data from our sheep studies of $P$ aeruginosa pneumonia which suggested that DMSO lowered the efficacy of MSC on oxygenation. ${ }^{6}$ For future trials, we will obtain all MSCs from the University of Minnesota and will use those showing 100\% viability before distribution. We will also eliminate the wash step for removal of DMSO.

Some questions remain about the biology and clinical testing of MSCs harvested from bone marrow, umbilical cord, or adipose tissue. An important issue is the uncertainty about the effects of live and dead MSCs. ${ }^{27}$ Some data suggest that live and dead human cells evoke similar responses in mouse lungs and in some experimental studies, dead cells have produced favourable immune responses after being phagocytised by monocytes or macrophages. ${ }^{27}$ More research is needed to optimise the methods for MSC cryopreservation, thawing, and production of MSCs for clinical trials.

Several other therapy-related issues deserve comment. The optimum delivery route for MSC therapy for ARDS has not been established. In our preclinical studies we used intrabronchial, intratracheal, and intravenous routes, all of which were associated with efficacy. ${ }^{7,9}$ Because of an excellent safety track record of MSC administration by the intravenous route in other clinical disorders, the FDA advised us to test the intravenous route in our safety trials of MSCs for ARDS, as this would probably be more reasonable for eventual regulatory approval than administration by the intrabronchial route. We aimed to give MSC therapy in the exudative phase of ARDS. In one study, 74 (90\%) of 82 patients who died within 7 days of diagnosis of ARDS had post-mortem evidence of the exudative phase of lung injury and minimal evidence of fibrosis. ${ }^{10}$ Because we treated patients within 7 days of ARDS diagnosis, it seems likely that administration of MSCs during the exudative phase was achieved.

We screened a large number of patients for this trial to be able to recruit $60(6 \%)$. In multiple phase 3 trials done by the National Heart, Lung, and Blood Institute ARDS Network, approximately $10 \%$ of screened populations were enrolled. We have noted differences between observational studies and clinical trials of ARDS in the selection of patients and the presence of comorbidities relative to the higher mortality reported. ${ }^{28}$ The results of clinical trials in which screening includes several exclusion criteria, as in this trial, are not necessarily generalisable to the broader unselected population of ARDS patients. If such trials test supportive care interventions, such as low tidal volume or fluid conservative therapy, the findings might have broader relevance, ${ }^{2}$ but, for pharmacological and cell-based therapies, extrapolation of the findings might not be appropriate.

Further limitations of this trial were that the sample size was too small to reliably assess efficacy. For example, the Billiards trial, ${ }^{29}$ which tested a strategy of reduced tidal volume ventilation against an established strategy in 52 patients with ARDS, showed no difference in clinical outcomes between groups. By contrast, the larger phase 3 ARMA trial of 861 patients ${ }^{30}$ showed reduced in-hospital mortality with low tidal volume ventilation. The findings of phase 2 trials in ARDS, in which CIs are often wide, therefore, have limited 
generalisability. ${ }^{31}$ Additionally, some analyses in this trial were done post hoc without adjustment for multiple comparisons.

This randomised, double-blind, phase $2 \mathrm{a}$ trial of treatment with human bone-marrow-derived MSCs for moderate to severe ARDS in critically ill patients, including patients with vasopressor-dependent shock at baseline, showed no infusion-related haemodynamic or respiratory adverse events. The patients in the MSC group had numerically higher disease severity scores than those in the placebo group at baseline, but mortality did not differ significantly between groups. Concentrations of angiopoietin 2 in plasma were significantly reduced at $6 \mathrm{~h}$ in MSC recipients. An important finding was that MSC viability had a significant viability-dependent effect on angiopoietin- 2 concentrations. We anticipate being able to avoid wide variability in MSC viability in future trials by changes to product preparation methods. Larger trials will be needed to test the efficacy of MSCs for ARDS. We are planning a phase $2 \mathrm{~b}$ trial in 120 patients that will be powered to detect efficacy for respiratory endpoints, with oxygenation index as the primary endpoint.

\section{Supplementary Material}

Refer to Web version on PubMed Central for supplementary material.

\section{Acknowledgments}

This work was funded by the National Heart, Lung, and Blood Institute Production Assistance for Cellular Therapies Program (U01 HL108713 and HHSN26820100008C). We thank the clinical coordinators and bonemarrow transplant staff at the medical centres: Faye Pais, Brian Daniel, Annika Belzer (coordinators), and Xiaohui Fang (laboratory investigator), University of California San Francisco; Sarah F Rapport (coordinator) and Misty DeRiggi (bone-marrow-transplantation laboratory lead technician), University of Pittsburgh Medical Center; Emily Robart, Luke Herren (coordinators), Beth Besecker (co-investigator), Hillary Bradbury, and Beth Daneault (bonemarrow-transplantation laboratory), Ohio State University Medical Center; Rosemary Vojnik (coordinator) and Mary McLeod (bone-marrow-transplantation laboratory), Stanford University; Kelsey Brait (coordinator), Massachusetts General Hospital; and Diane Kadidlo, (technical supervisor) and Darin Sumstad (technical specialist), University of Minnesota Program Assisted Cellular Therapeutics and Translational Development Team of the Cell Therapy Laboratory of University of Minnesota Medical Center.

Declaration of interests

MAM has received grants from Bayer Pharmaceuticals and GlaxoSmithKline, personal fees from Boehringer Ingelheim, Cerus Therapeutics, CSL Berhing, Quark Pharmaceuticals, Roche-Genentec, and Thesan Pharmaceuticals. CSC has received grants and personal fees from Bayer and GlaxoSmithKline, personal fees from Boehringer Ingelheim, CSL Behring, Prometic, and Roche/Genentech. EKB has received personal fees from Merck. BJM has received grants from Bayer Pharmaceuticals and personal fees from Vapotherm. KDL has received personal fees from Achaogen, Durect, Potrero Medical, Quark, Theravance, and Z S Pharma, and other funding or awards from Amgen. The other authors declare no competing interests.

\section{References}

1. Matthay MA, Ware LB, Zimmerman GA. The acute respiratory distress syndrome. J Clin Invest 2012; 122: 2731-40. [PubMed: 22850883]

2. Thompson BT, Chambers RC, Liu KD. Acute respiratory distress syndrome. N Engl J Med 2017; 377: 562-72. [PubMed: 28792873]

3. Bellani G, Laffey JG, Pham T, et al. Epidemiology, patterns of care, and mortality for patients with acute respiratory distress syndrome in intensive care units in 50 countries. JAMA 2016; 315: 788800. [PubMed: 26903337] 
4. Matthay MA, Pati S, Lee JW. Concise review: mesenchymal stem (stromal) cells: biology and preclinical evidence for therapeutic potential for organ dysfunction following trauma or sepsis. Stem Cells 2017; 35: 316-24. [PubMed: 27888550]

5. Walter J, Ware LB, Matthay MA. Mesenchymal stem cells: mechanisms of potential therapeutic benefit in ARDS and sepsis. Lancet Respir Med 2014; 2: 1016-26. [PubMed: 25465643]

6. Asmussen S, Ito H, Traber DL, et al. Human mesenchymal stem cells reduce the severity of acute lung injury in a sheep model of bacterial pneumonia. Thorax 2014; 69: 819-25. [PubMed: 24891325]

7. Lee JW, Fang X, Gupta N, Serikov V, Matthay MA. Allogeneic human mesenchymal stem cells for treatment of $E$. coli endotoxin-induced acute lung injury in the ex vivo perfused human lung. Proc Natl Acad Sci USA 2009; 106: 16357-62. [PubMed: 19721001]

8. Lee JW, Krasnodembskaya A, McKenna DH, Song Y, Abbott J, Matthay MA. Therapeutic effects of human mesenchymal stem cells in ex vivo human lungs injured with live bacteria. Am J Respir Crit Care Med 2013; 187: 751-60. [PubMed: 23292883]

9. Laffey JG, Matthay MA. Fifty years of research in ARDS. Cell-based therapy for acute respiratory distress syndrome. biology and potential therapeutic value. Am J Respir Crit Care Med 2017; 196: 266-73. [PubMed: 28306336]

10. Thille AW, Esteban A, Fernandez-Segoviano P, et al. Chronology of histological lesions in acute respiratory distress syndrome with diffuse alveolar damage: a prospective cohort study of clinical autopsies. Lancet Respir Med 2013; 1: 395-401. [PubMed: 24429204]

11. Force ADT, Ranieri VM, Rubenfeld GD, et al. Acute respiratory distress syndrome: the Berlin Definition. JAMA 2012; 307: 2526-33. [PubMed: 22797452]

12. Matthay MA, McAuley DF, Ware LB. Clinical trials in acute respiratory distress syndrome: challenges and opportunities. Lancet Respir Med 2017; 5: 524-34. [PubMed: 28664851]

13. Wilson JG, Liu KD, Zhuo H, et al. Mesenchymal stem (stromal) cells for treatment of ARDS: a phase 1 clinical trial. Lancet Respir Med 2015; 3: 24-32. [PubMed: 25529339]

14. NHLBI ARDS Network. NIH NHLBI ARDS Clinical Network mechanical ventilation protocol summary. http://www.ardsnet.org/files/ventilator_protocol_2008-07.pdf (accessed Oct 29, 2018).

15. Liu KD, Wilson JG, Zhuo H, et al. Design and implementation of the START (STem cells for ARDS Treatment) trial, a phase 1/2 trial of human mesenchymal stem/stromal cells for the treatment of moderate-severe acute respiratory distress syndrome. Ann Intensive Care 2014; 4: 22. [PubMed: 25593740]

16. Rosenberg AL. Recent innovations in intensive care unit risk-prediction models. Curr Opin Crit Care 2002; 8: 321-30. [PubMed: 12386493]

17. Wiedemann HP, Wheeler AP, Bernard GR, et al. Comparison of two fluid-management strategies in acute lung injury. N Engl J Med 2006; 354: 2564-75. [PubMed: 16714767]

18. Seeley E, McAuley DF, Eisner M, Miletin M, Matthay MA, Kallet RH. Predictors of mortality in acute lung injury during the era of lung protective ventilation. Thorax 2008; 63: 994-98. [PubMed: 18566110]

19. Murray JF, Matthay MA, Luce JM, Flick MR. An expanded definition of the adult respiratory distress syndrome. Am Rev Respir Dis 1988; 138: 720-23. [PubMed: 3202424]

20. Bhandari V, Choo-Wing R, Lee CG, et al. Hyperoxia causes angiopoietin 2-mediated acute lung injury and necrotic cell death. Nat Med 2006; 12: 1286-93. [PubMed: 17086189]

21. Parikh SM. Angiopoietins and Tie2 in vascular inflammation. Curr Opin Hematol 2017; 24: 43238. [PubMed: 28582314]

22. Agrawal A, Matthay MA, Kangelaris KN, et al. Plasma angiopoietin-2 predicts the onset of acute lung injury in critically ill patients. Am J Respir Crit Care Med 2013; 187: 736-42. [PubMed: 23328529]

23. Calfee CS, Gallagher D, Abbott J, Thompson BT, Matthay MA, Network NA. Plasma angiopoietin-2 in clinical acute lung injury: prognostic and pathogenetic significance. Crit Care Med 2012; 40: 1731-37. [PubMed: 22610178]

24. Zinter MS, Spicer A, Orwoll BO, et al. Plasma angiopoietin-2 outperforms other markers of endothelial injury in prognosticating pediatric ARDS mortality. Am J Physiol Lung Cell Mol Physiol 2016; 310: L224-31. [PubMed: 26660787] 
25. Gutbier B, Neuhauss AK, Reppe K, et al. Prognostic and pathogenic role of angiopoietin-1 and -2 in pneumonia. Am J Respir Crit Care Med 2018; 198: 220-31. [PubMed: 29447449]

26. Kaplan A, Sackett K, Sumstad D, Kadidlo D, McKenna DH. Impact of starting material (fresh versus cryopreserved marrow) on mesenchymal stem cell culture. Transfusion 2017; 57: 2216-19. [PubMed: 28653392]

27. Galipeau J, Sensebe L. Mesenchymal stromal cells: clinical challenges and therapeutic opportunities. Cell Stem Cell 2018; 22: 824-33. [PubMed: 29859173]

28. Pais FM, Sinha P, Liu KD, Matthay MA. Influence of clinical factors and exclusion criteria on mortality in ards observational studies and randomized controlled trials. Respir Care 2018; 63: 1060-69. [PubMed: 29991643]

29. Brower RG, Shanholtz CB, Fessler HE, et al. Prospective, randomized, controlled clinical trial comparing traditional versus reduced tidal volume ventilation in acute respiratory distress syndrome patients. Crit Care Med 1999; 27: 1492-98. [PubMed: 10470755]

30. Brower RG, Matthay MA, Morris A, et al. Ventilation with lower tidal volumes as compared with traditional tidal volumes for acute lung injury and the acute respiratory distress syndrome. $\mathrm{N}$ Engl J Med 2000; 342: 1301-08. [PubMed: 10793162]

31. Rubenfeld GD, Abraham E. When is a negative phase II trial truly negative? Am J Respir Crit Care Med 2008; 178: 554-55. [PubMed: 18755930] 


\section{Research in context}

\section{Evidence before this study}

We searched PubMed for studies published between Jan 1, 2003, and Jan 1, 2013, with the search string "(acute respiratory distress syndrome OR acute lung injury) AND (marrow-derived mesenchymal stem cells) AND ('2003/01/01' [Date -Publication]: '2013/01/01' [Date -Publication])" and no language restrictions. Preclinical studies of ARDS in small and large animals and perfused ex-vivo human lung preparations have shown therapeutic benefits after treatment with bone-marrow-derived mesenchymal stromal cells (MSCs). Based on these findings, clinical studies were done to assess the effects of MSCs to treat ARDS. One dose-escalation trial of 1, 5, and $10 \times 10^{6} \mathrm{MSCs} / \mathrm{kg}$ predicted bodyweight in nine patients with moderate to severe ARDS indicated that the highest dose could be well tolerated. We obtained regulatory approval from the US Food and Drug Administration to do a phase 2a multicentre, randomised, double-blind trial of MSCs to assess safety.

\section{Added value of this study}

In patients with moderate to severe ARDS based on prespecified haemodynamic and respiratory parameters, MSC therapy was well tolerated. Clinical outcomes did not differ significantly between groups, but post-hoc analyses showed a trend for improvement in oxygenation index in the MSC group. Post-thaw viability of MSCs emerged as a potentially important factor in the biological and clinical effects of this treatment for ARDS.

\section{Implications of all the available evidence}

Our results support the finding that single-dose intravenous MSC therapy is well tolerated in patients with moderate to severe ARDS. Measurement of biomarkers suggested that endothelial injury is significantly reduced, and that the effect is associated with higher viability of MSCs after thawing. Further well powered trials are needed to assess efficacy. 


\section{Panel: Prespecified infusion-associated adverse events}

\section{Within $6 \mathrm{~h}$ of study product infusion}

- Increase in vasopressor dose to the following values or higher:

- $\quad$ Norepinephrine $10 \mu \mathrm{g} / \mathrm{min}$

- $\quad$ Phenylephrine $100 \mu \mathrm{g} / \mathrm{min}$

- $\quad$ Dopamine $10 \mu \mathrm{g} / \mathrm{kg}$ per min

- $\quad$ Epinephrine $0 \cdot 1 \mu \mathrm{g} / \mathrm{kg}$ per min or addition of a third vasopressor

- New ventricular tachycardia, ventricular fibrillation or asystole

- New cardiac arrhythmia requiring cardioversion

- Hypoxaemia requiring an increase in $\mathrm{FiO}_{2}$ of 0.2 or more and an increase in PEEP of 5.0 or more to maintain $\mathrm{SpO}_{2}$ in the target range of $88-95 \%$

- Clinical scenario consistent with transfusion incompatibility or transfusionrelated infection (eg, urticaria, new bronchospasm)

\section{Within $24 \mathrm{~h}$ of study product infusion}

- $\quad$ Any cardiac arrest or death

$\mathrm{FiO}_{2}=$ fractional concentration of oxygen. $\mathrm{PEEP}=$ positive end-expiratory pressure.

$\mathrm{SpO}_{2}=$ peripheral capillary oxygen saturation. 


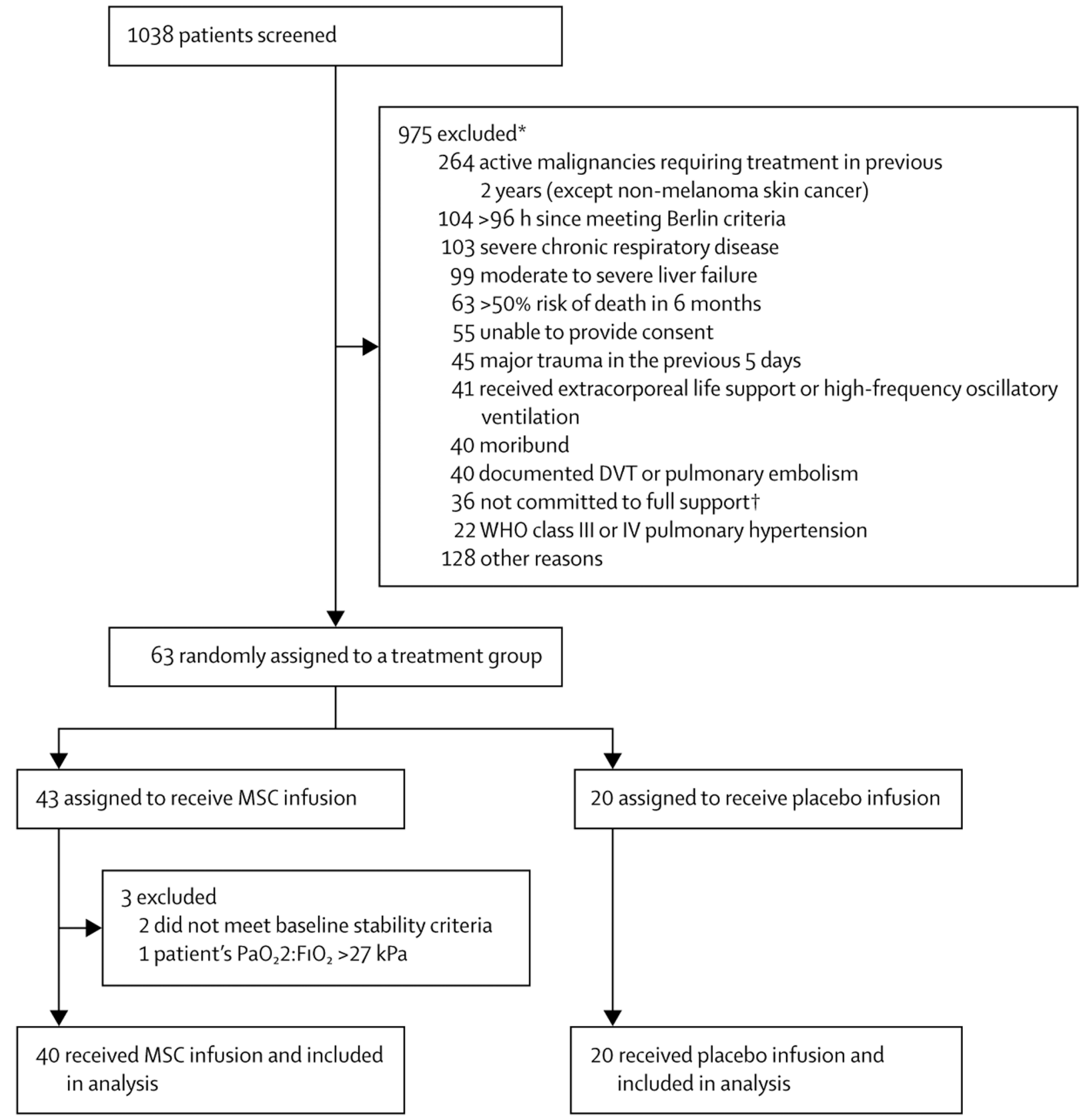

Figure 1: Trial profile

DVT=deep-vein thrombosis. MSC=mesenchymal stromal cell. $\mathrm{PaO}_{2}: \mathrm{FiO}_{2}=$ ratio of partial pressure of oxygen to fractional inspired oxygen. *Numbers total more than 975 because some patients met multiple exclusion criteria. $†$ Do not resuscitate or limit on life support order in place. 


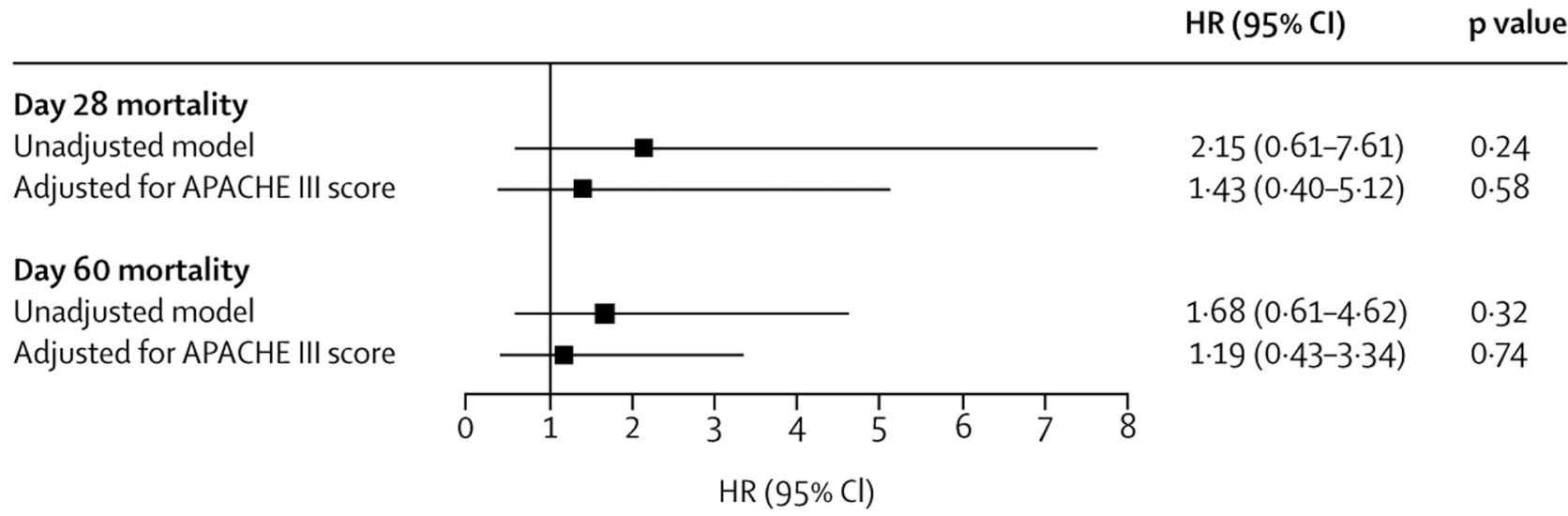

Figure 2: Mortality to day 28 and day 60 for mesenchymal stromal cell therapy versus placebo APACHE III=Acute Physiology and Chronic Health Evaluation III score; HR=hazard ratio. 

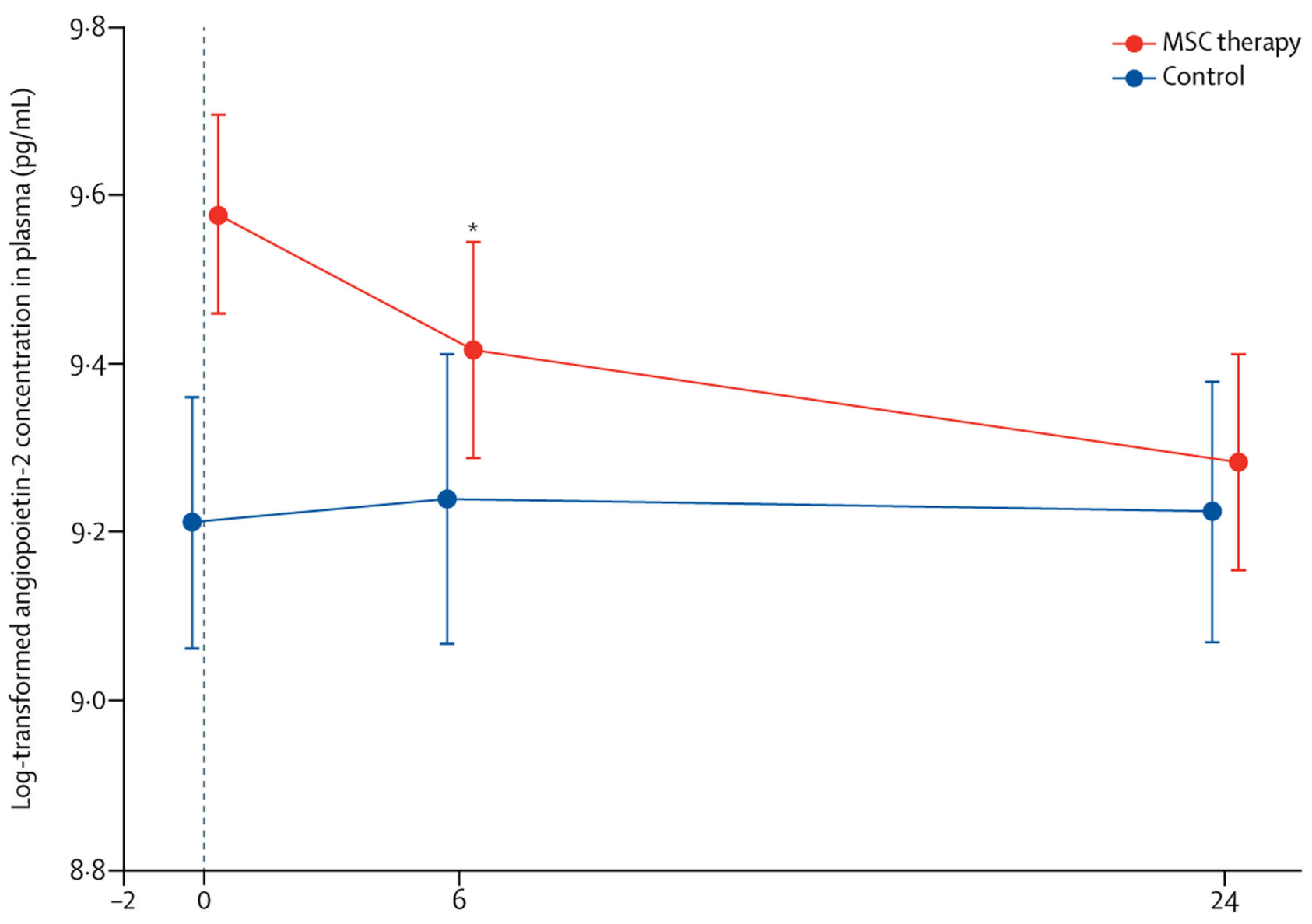

Time after infusion (h)

Figure 3: Changes in angiopoietin 2 concentrations in plasma from baseline to 6 and $24 \mathrm{~h}$ Data are mean (SE) after log transformation. $\mathrm{MSC}=$ mesenchymal stromal cell. $* \mathrm{p}=0.005$ compared with placebo. 


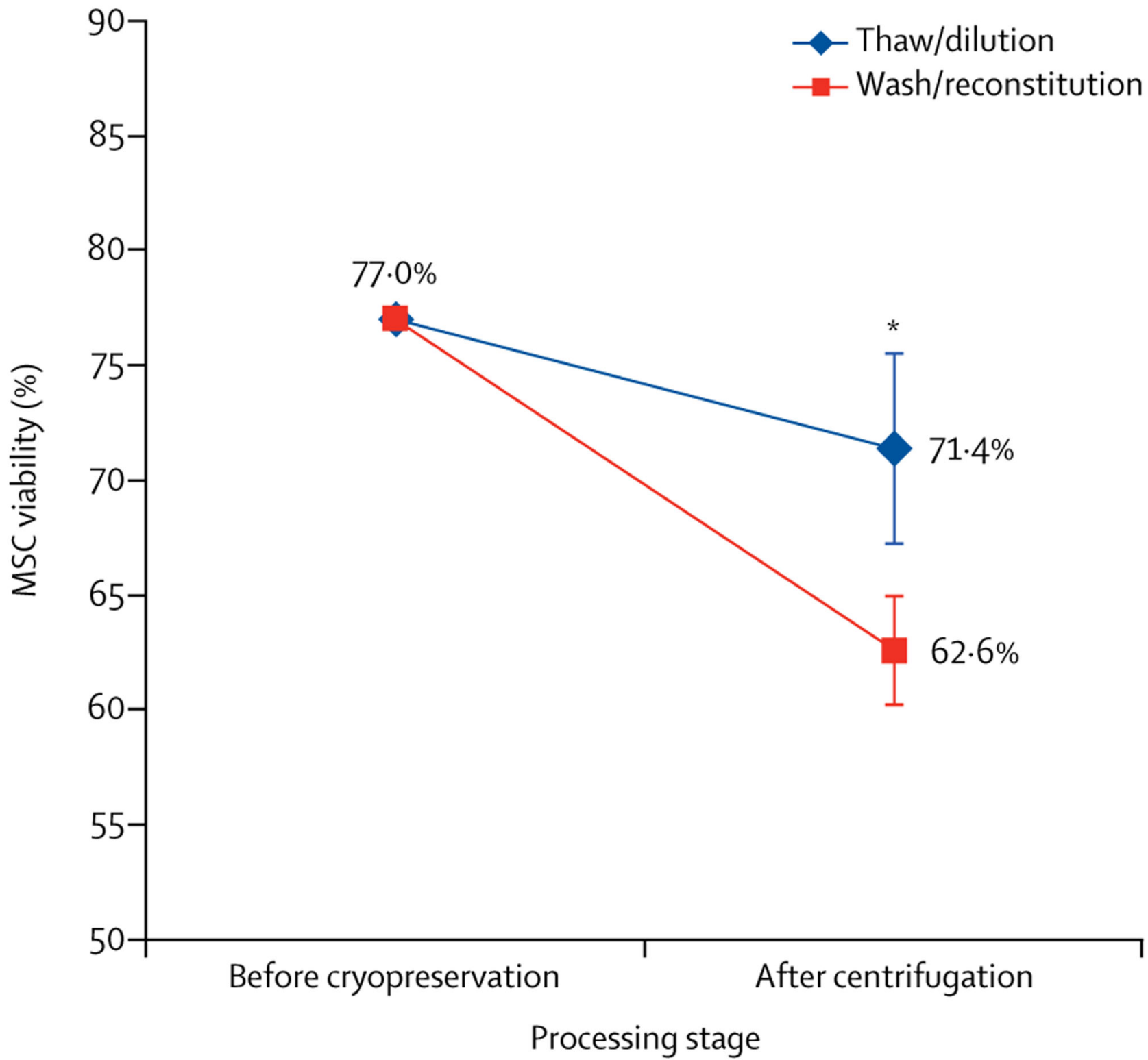

Figure 4: MSC viability measured after simple thawing and dilution versus after cell wash and reconstitution

Data are mean (SD) based on viability measurements at 0, 30, 60, 120, and 180 min after removal from cryopreservation. $\mathrm{MSC}=$ mesenchymal stromal cell. ${ }^{*} \mathrm{p}=0.004$ compared with before cryopreservation. 
Table 1:

Baseline characteristics

\begin{tabular}{|c|c|c|}
\hline & Mesenchymal stromal cell group $(n=40)$ & Placebo group $(n=20)$ \\
\hline \multicolumn{3}{|l|}{ Patients' characteristics } \\
\hline Age (years) & $55(17)$ & $55(20)$ \\
\hline Men/women & $23(58 \%) / 17(42 \%)$ & $10(50 \%) / 10(50 \%)$ \\
\hline \multicolumn{3}{|l|}{ Cause of ARDS } \\
\hline Sepsis with pneumonia & $19(48 \%)$ & $12(60 \%)$ \\
\hline Sepsis without pneumonia & $5(13 \%)$ & $2(10 \%)$ \\
\hline Pneumonia without sepsis & $11(28 \%)$ & $5(25 \%)$ \\
\hline Aspiration only & $4(10 \%)$ & $1(5 \%)$ \\
\hline Other & $1(3 \%)$ & 0 \\
\hline Arterial pressure $(\mathrm{mm} \mathrm{Hg})$ & $75(10)$ & $76(9)$ \\
\hline Taking vasopressors at the time of infusion & $24(60 \%)$ & $9(45 \%)$ \\
\hline SOFA score & $8 \cdot 1(3 \cdot 3)$ & $6 \cdot 9(2 \cdot 7)$ \\
\hline APACHE III score & $104(31)$ & $89(33)$ \\
\hline \multicolumn{3}{|l|}{ Respiratory characteristics } \\
\hline Minute ventilation (L/min) & $11 \cdot 1(3 \cdot 2)$ & $9 \cdot 6(2 \cdot 4)$ \\
\hline Respiratory rate (breaths/min) & $27 \cdot 8(6 \cdot 6)$ & $24 \cdot 5(6 \cdot 3)$ \\
\hline Tidal volume (mL/kg PBW) & $6 \cdot 3(0 \cdot 9)$ & $6 \cdot 1(0 \cdot 7)$ \\
\hline Mean airway pressure $\left(\mathrm{cm} \mathrm{H}_{2} \mathrm{O}\right)$ & $17 \cdot 8(4 \cdot 9)$ & $16 \cdot 4(3 \cdot 6)$ \\
\hline Plateau airway pressure $\left(\mathrm{cm} \mathrm{H}_{2} \mathrm{O}\right)$ & $26 \cdot 4(5 \cdot 7)$ & $23 \cdot 7(5 \cdot 1)$ \\
\hline PEEP $\left(\mathrm{cm} \mathrm{H}_{2} \mathrm{O}\right)$ & $12 \cdot 4(3 \cdot 7)$ & $10 \cdot 8(2 \cdot 6)$ \\
\hline Driving pressure $\left(\mathrm{cm} \mathrm{H}_{2} \mathrm{O}\right)$ & $14 \cdot 0(4 \cdot 1)$ & $12 \cdot 5(4 \cdot 3)$ \\
\hline $\mathrm{PaO}_{2}: \mathrm{FiO}_{2}(\mathrm{kPa})$ & $18 \cdot 1(4 \cdot 3)$ & $19 \cdot 1(5 \cdot 2)$ \\
\hline Oxygenation index $(\mathrm{kPa})^{*}$ & $98 \cdot 7(78 \cdot 1-123 \cdot 3)$ & $95 \cdot 6(71 \cdot 6-113 \cdot 8)$ \\
\hline Lung injury score & $3 \cdot 1(00 \cdot 4)$ & $3 \cdot 0(0 \cdot 5)$ \\
\hline \multicolumn{3}{|l|}{ Ventilation mode } \\
\hline Volume control & $37(93 \%)$ & $17(85 \%)$ \\
\hline Pressure-regulated volume control & $1(3 \%)$ & $2(10 \%)$ \\
\hline Other & $2(5 \%)$ & $1(5 \%)$ \\
\hline
\end{tabular}

Data are $\mathrm{n}(\%)$, mean $(\mathrm{SD})$, or median (IQR); percentage values might not sum 100\% due to rounding. ARDS=acute respiratory distress syndrome; SOFA=sequential organ failure assessment. APACHE III=Acute Physiology and Chronic Health Evaluation III score. PBW=predicted bodyweight. $\mathrm{PEEP}=$ positive end-expiratory pressure. $\mathrm{PaO}_{2}: \mathrm{FiO}_{2}=$ ratio of partial pressure of oxygen to fractional inspired oxygen. Oxygenation index $=\mathrm{FiO} 2 \times$ mean airway pressure / $\mathrm{PaO}_{2}$.

* Unavailable for three patients in the mesenchymal stromal cell group and one in the placebo group because mean airway pressure was not recorded. 
Table 2:

Main clinical outcomes

\begin{tabular}{|c|c|c|c|c|}
\hline & $\begin{array}{l}\text { Mesenchymal stromal cell } \\
\text { group }(\mathbf{n}=40)\end{array}$ & Placebo group $(n=20)$ & $\begin{array}{l}\text { Odds ratio }{ }^{*} \text { or median } \\
\text { difference }(95 \% \mathrm{CI})\end{array}$ & p value \\
\hline Mortality to day 28 & $12(30 \%)$ & $3(15 \%)$ & $2 \cdot 4(0 \cdot 5$ to $15 \cdot 1)$ & $0 \cdot 34$ \\
\hline Mortality to day 60 & $15(38 \%)$ & $5(25 \%)$ & $1.8(0 \cdot 5$ to $7 \cdot 6)$ & $0 \cdot 40$ \\
\hline $\begin{array}{l}\text { Number of ventilator-free days to } \\
\text { day } 28\end{array}$ & $2(0$ to 23$)$ & 17 ( 0 to 24$)$ & $0(-12$ to 0$)$ & $0 \cdot 28$ \\
\hline $\begin{array}{l}\text { Number of ICU-free days to day } \\
28\end{array}$ & $2(0$ to 19$)$ & 14 (7 to 20$)$ & $-5(-11$ to 0$)$ & $0 \cdot 05$ \\
\hline \multicolumn{5}{|c|}{ Number of organ-failure-free days to day 28} \\
\hline Cardiovascular & 18 (7 to 24$)$ & 24 (19 to 27$)$ & $-4(-10$ to 0$)$ & $0 \cdot 09$ \\
\hline Coagulation & $28(17$ to 28$)$ & 28 (28 to 28$)$ & $0(-3$ to 0$)$ & $0 \cdot 10$ \\
\hline Hepatic & 28 (16 to 28$)$ & $28(25$ to 28$)$ & $0(0$ to 0$)$ & $0 \cdot 43$ \\
\hline Renal & 28 (7 to 28$)$ & $28(25$ to 28$)$ & $0(-5$ to 0$)$ & $0 \cdot 25$ \\
\hline Non-pulmonary organs & 12 (2 to 24$)$ & $22(13$ to 25$)$ & $-4(-13$ to 0$)$ & $0 \cdot 10$ \\
\hline $\begin{array}{l}\text { Duration of ventilation in } \\
\text { survivors to day } 28 \text { (days) }\end{array}$ & 12 (4 to 24$)$ & $8(4$ to 15$)$ & $2(-3$ to 10$)$ & $0 \cdot 51$ \\
\hline
\end{tabular}

Data are n (\%), odds ratio (95\% CI), or median (IQR) with median difference (95\% CI calculated by Hodges-Lehmann method). Fisher's exact test was used to compare categorical variables, and Mann-Whitney $U$ test for continuous variables. ICU=intensive-care unit.

* Calculated for mortality. 


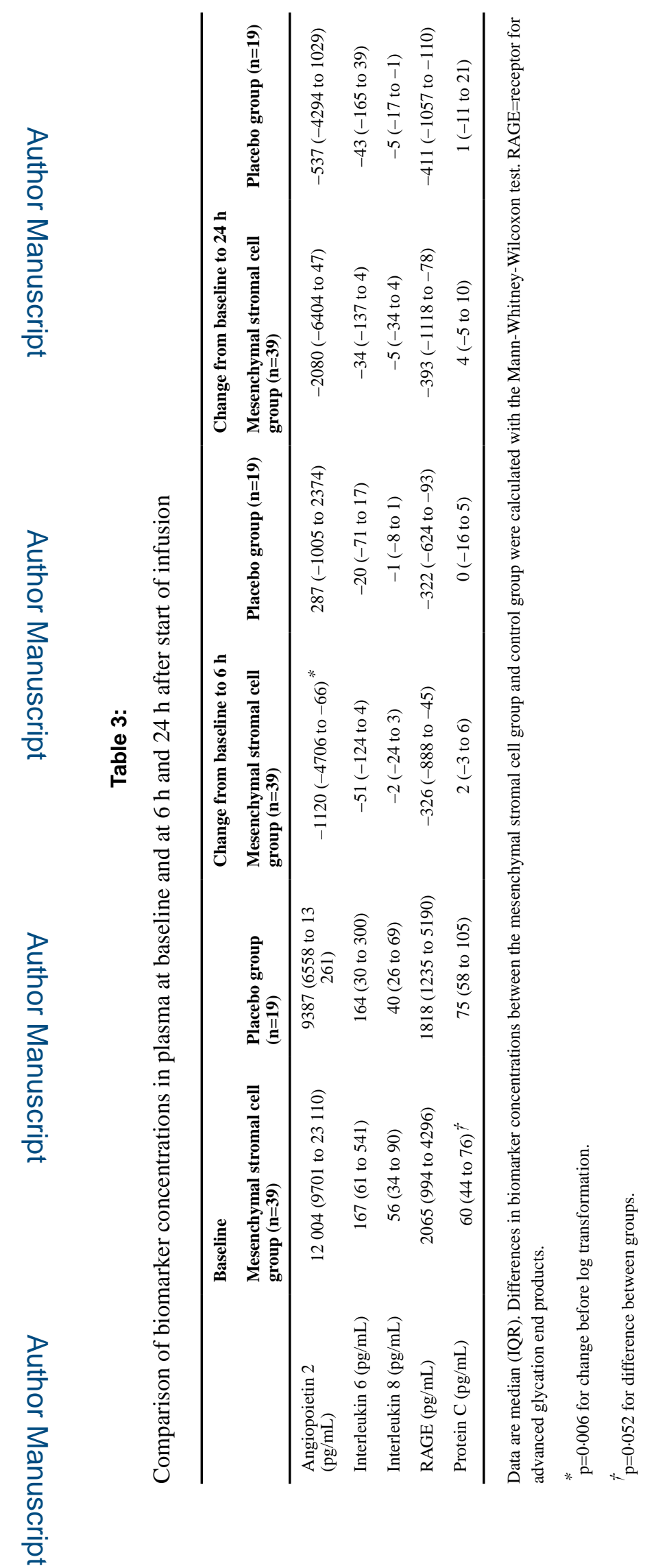

Lancet Respir Med. Author manuscript; available in PMC 2020 October 30. 